\title{
Superlubricity of Graphite
}

\author{
Martin Dienwiebel, ${ }^{*}$ Gertjan S. Verhoeven, Namboodiri Pradeep, and Joost W. M. Frenken ${ }^{\dagger}$ \\ Kamerlingh Onnes Laboratory, Leiden University, P.O. Box 9504, 2300 RA Leiden, The Netherlands \\ Jennifer A. Heimberg \\ FOM-Institute for Atomic and Molecular Physics, Kruislaan 407, 1098 SJ Amsterdam, The Netherlands
}

Henny W. Zandbergen

Laboratory of Materials Science, Delft University of Technology, Rotterdamseweg 137, 2628 AL Delft, The Netherlands

(Received 31 July 2003; published 24 March 2004)

\begin{abstract}
Using a home-built frictional force microscope that is able to detect forces in three dimensions with a lateral force resolution down to $15 \mathrm{pN}$, we have studied the energy dissipation between a tungsten tip sliding over a graphite surface in dry contact. By measuring atomic-scale friction as a function of the rotational angle between two contacting bodies, we show that the origin of the ultralow friction of graphite lies in the incommensurability between rotated graphite layers, an effect proposed under the name of "superlubricity" [M. Hirano and K. Shinjo, Phys. Rev. B 41, 11837 (1990)].
\end{abstract}

DOI: 10.1103/PhysRevLett.92.126101

Graphite and other lamellar solids such as molybdenum disulphide $\left(\mathrm{MoS}_{2}\right)$ are known to be good solid lubricants and, thus, are widely used in practical applications. The low-friction behavior is traditionally ascribed to the low resistance to shear between neighboring atomic layers of these materials [1]. The slipperiness of graphite, responsible for its excellent lubrication properties, is also observed in frictional force microscopy (FFM) experiments [2-6], in which friction coefficients as low as 0.001 have been found. In these experiments, a wide variety of atomically sharp tips have been sheared over graphite surface, while the lateral forces were recorded with $\mathrm{nN}$ precision. These experiments also revealed that the scanning tip in FFM experiments on graphite performs a so-called "stick-slip" movement, where the tip jumps discontinuously over single lattice spacings. This leads to variations in the lateral force with the periodicity of the graphite lattice. A simple classical mechanics model [7], the socalled Tomlinson model, where a pointlike tip is moved along in a two-dimensional sinusoidal potential, reproduced the experimental lateral force maps of the graphite surface quite well $[8,9]$. However, the normal forces that had to be used in the simulations had to be 1 to 2 orders of magnitude lower than in the experiments in order to fit the experiments [10]. It has therefore been proposed that in these experiments the contact might not consist of a single atom but of a large, but finite number of atoms, performing a collective atomic-scale slip-stick motion.

In scanning probe microscopy experiments on graphite, it is known that thin, flat flakes of graphite, parallel to the natural lattice planes of graphite, are frequently transferred to the scanning tip (e.g., [11]), thus providing a multiatom contact. Based on this, a natural explanation for the low-friction behavior of graphite might be superlubricity. This conceptually simple phenomenon has been proposed to occur when two parallel surfaces slide over
PACS numbers: 68.35.Af, 07.79.Sp, 62.20.Qp, 68.37.Ps

each other in incommensurate contact [12-14]. In such a geometry, the lattice mismatch can prevent collective stick-slip motion of all atoms in the contact together, and thus the kinetic friction force can be vanishingly small $[12,13]$. A few earlier experiments provided indications of superlubricity. Hirano et al. [15] found a modest reduction in friction between two mica sheets from $8 \times 10^{-4}$ to $2 \times 10^{-4} \mathrm{~N}$, caused by rotating the two sheets with respect to each other. In a modified scanning tunneling microscope experiment [16], the same authors claimed the observation of superlubricity between a tungsten tip and a $\mathrm{Si}(001)$ sample. Ko and Gellman measured the friction force as a function of the misfit angle between two $\mathrm{Ni}(100)$ crystal surfaces [17]. They found lowered friction for certain angles, but they ascribed this to easy shearing along the preferred slip planes in the bulk. Although superlubricity had been proposed already in 1990, it has found little attention, in spite of the promise that it can dramatically reduce friction in dry, unlubricated contacts, which would make it highly relevant for a wide variety of applications, such as, e.g., nanoelectromechanical systems.

In order to identify and study superlubricity, we have developed a novel frictional force microscope, which allows quantitative tracking of the forces on the scanning tip in three directions, with a resolution in the lateral forces, down to $15 \mathrm{pN}$. The key feature of our microscope is a dedicated friction force sensor [18], the Tribolever (see Fig. 1). It combines low and symmetric spring constants in the two lateral directions with a high stiffness in the normal, in order to avoid "snap to contact." We determined the lateral spring constants of the Tribolever to be $k_{x, y}^{\text {Tribolever }}=5.75 \pm 0.15 \mathrm{~N} / \mathrm{m}$ and the normal spring constant of $26 \pm 1 \mathrm{~N} / \mathrm{m}$, using the "added-mass method" by Cleveland et al. [19]. A tungsten tip was glued into the Tribolever using silver epoxy, such that it 


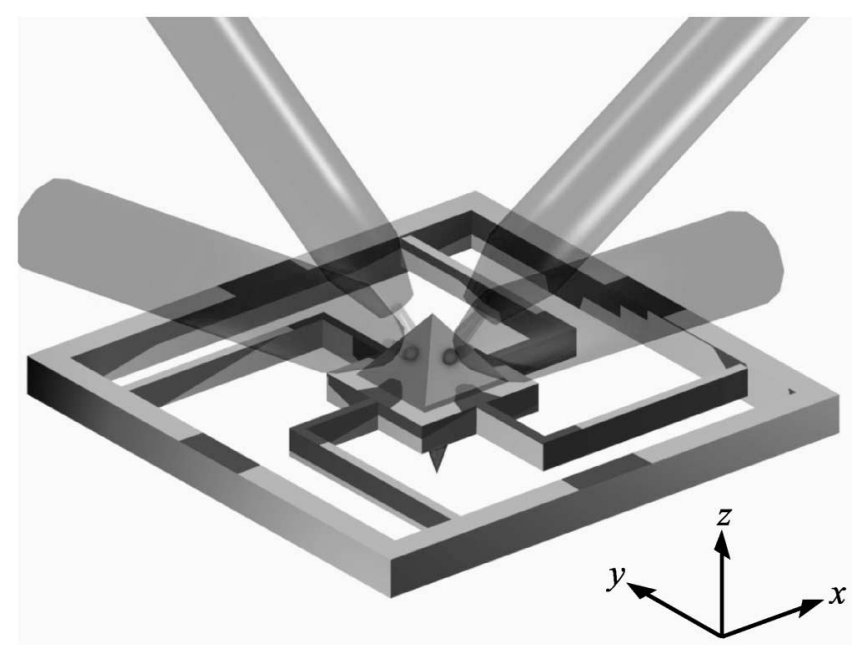

FIG. 1. Model of the microfabricated silicon Tribolever together with the four glass fibers that are used to detect the three-dimensional motion of the scanning tip. Four legs, placed symmetrically around a central pyramid (which acts as the mirrors for the interferometers), form a set of equally sensitive springs in the $X$ and $Y$ directions. The tungsten tip, which is pointing down, is mounted in a hole in the pyramid.

extended about 50 to $60 \mu \mathrm{m}$ out of the device. The displacements of the Tribolever tip are monitored using four all-glass-fiber interferometers (Fig. 1). The instrument can rotate the sample to change the relative orientation between the tip and sample lattices.

We performed measurements on highly oriented pyrolitic graphite grade ZYA, which possesses a lateral grain size of up to $10 \mathrm{~mm}$. The sample was cut to a size of $2 \mathrm{~mm} \times 2 \mathrm{~mm}$ and, therefore, the grade ZYA sample consisted probably of a single graphite crystal. The sample was freshly cleaved using scotch tape and then mounted in the FFM inside a small chamber, which was continuously flushed with dry nitrogen. The measurements were performed at relative humidities of $(1-10) \% \pm 1 \%$ RH. The scanning speed in the experiments was $30 \mathrm{~nm} / \mathrm{s}$. All force maps were recorded in the form of two-dimensional "images" with $512 \times 512$ pixels. The normal load $F_{N}$ was set using the feedback loop of the scan electronics. Zero normal load was defined as the load where the Tribolever was not bent in the normal direction. When the tip was scanned over the surface, we recorded the lateral forces in the scan direction and perpendicular to that, both during the forward and during the reverse scan lines. In contrast to a conventional AFM cantilever, with the Tribolever it is possible to choose any sliding direction in the measurement. One forward scan line and the subsequent reverse scan line together form a closed hysteresis loop, that is often referred to as the "friction loop."

We rotated the graphite sample in small steps with respect to the tungsten tip. For each orientation, we measured the lateral forces at a range of constant normal forces, between $+25 \mathrm{nN}$ and pull-off (typically $-22 \mathrm{nN}$ ). Over this normal force range, all measurements were completely reproducible and reversible, with no detectable damage to the surface or the tip. Figure 2(a) shows a typical force loop (one forward line and the subsequent backward line) of the force parallel to the sliding direction, at a normal force of $18 \mathrm{nN}$. The lateral force in Fig. 2(a) displays clearly resolved atomic-scale stick-slip sliding. Every time the force is sufficiently high, the tip slips over one lattice period of the graphite substrate. The area enclosed in the complete loop corresponds to the energy dissipated irreversibly during the loop, and the area divided by twice the loop width is the average, dissipative friction force, experienced by the tip, of $203 \pm 20 \mathrm{pN}$. Figure 2(b) shows the corresponding lateral force map of the lateral forces in the sliding direction, recorded in a two-dimensional friction scan.

The periodicity of the graphite substrate lattice can be recognized vaguely in the force variations. Although the normal and lateral forces used here are relatively low, the qualitative features of Figs. 2(a) and 2(b) are similar to those in many previously published FFM measurements on graphite. Figures 2(c) and 2(d) show FFM measurements under precisely the same conditions as those in Figs. 2(a) and 2(b), but with the graphite substrate rotated $12^{\circ}$ clockwise around an axis normal to the surface, and parallel to the tip. The rotation has caused the average friction force to reduce by more than 1 order of magnitude to $15.2 \pm 15 \mathrm{pN}$. This variation was completely reversible. Notice in Figs. 2(c) and 2(d) that the ultralow lateral force still displayed regular variations with the periodicity of the graphite substrate. Also rotating the sample away in the opposite direction reduced the average friction force to a near-zero value. This is shown in Figs. 2(e)
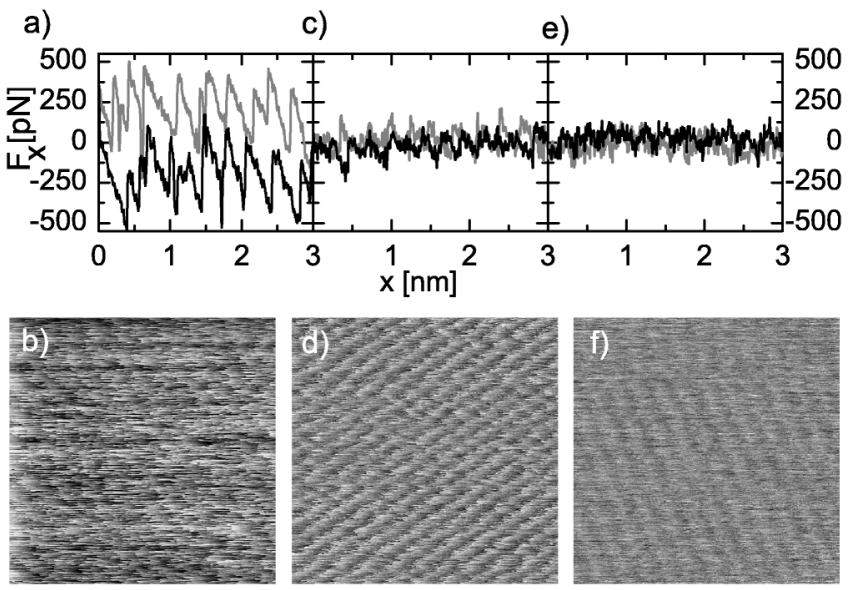

FIG. 2. Friction loops (black, forward; grey, reverse) and lateral force images (forward), measured along the scanning direction at tip-surface orientation angles $\Phi$ of $60^{\circ}$ (a),(b); $72^{\circ}$ (c),(d); $38^{\circ}$ (e),(f). Normal force $F_{N}=18 \mathrm{nN}$ (a)-(d) and $30.1 \mathrm{nN}$ (e),(f). Grey scale $590 \mathrm{pN}$ (b), $270 \mathrm{pN}$ (d), $265 \mathrm{pN}$ (f). Image size $3 \mathrm{~nm} \times 3 \mathrm{~nm}$. 
and 2(f) which were measured with the sample rotated $22^{\circ}$ anticlockwise from the initial rotational orientation of the sample. Figure 3 displays the average friction forces measured over a $100^{\circ}$ range of substrate rotation angles. We recognize two narrow angular regions with high friction, separated by a wide angular interval with nearly zero friction. The distance between the two friction peaks is $61^{\circ} \pm 2^{\circ}$, which corresponds well with the $60^{\circ}$ symmetry of individual atomic layers in the graphite lattice. In order to exclude instrumental artifacts, the friction values in Fig. 3 were always measured for the same sliding direction with respect to the substrate, which was possible by virtue of the complete equivalence of our sensor's force sensitivities in all lateral directions. However, we observed that the same variations in average friction force always occurred simultaneously for all sliding directions over the graphite lattice. The latticerelated orientational variation in the friction force and the near vanishing of friction for most orientations constitute firm evidence for superlubricity between the FFM tip and the graphite substrate. In the following, we provide strong arguments that the superlubricity has taken place between the graphite substrate and a graphite flake, attached to the tip. The presence of a flake between the tip and the substrate naturally explains the strong orientation dependence of the friction. At the two orientations corresponding to the friction peaks, the flake and substrate lattices have been perfectly aligned, while they have been incommensurate for the intermediate angles.

The flake scenario also explains the extremely weak dependence of the observed friction forces on the normal load [20] (not shown here). For other substrates, FFM measurements usually show a substantial dependence,

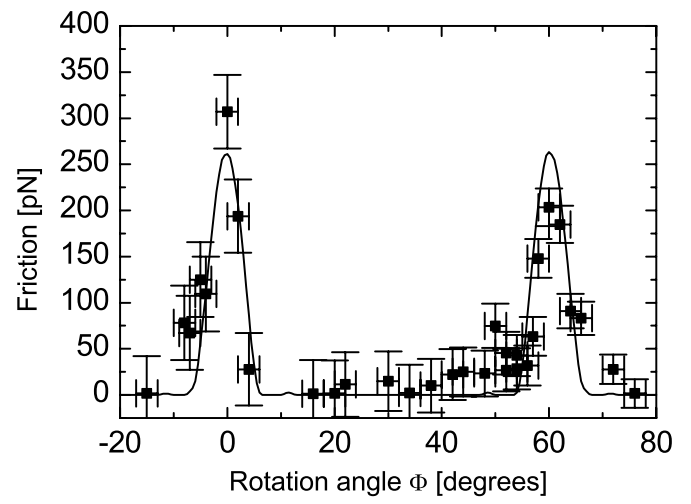

FIG. 3. Average friction force versus rotation angle of the graphite sample around an axis normal to the sample surface. Two narrow peaks of high friction were observed at 0 and $61^{\circ}$, respectively. Between these peaks a wide angular range with ultralow friction close to the detection limit of the instrument was found. The first peak has a maximum friction force of $306 \pm 40 \mathrm{pN}$, and the second peak has a maximum of $203 \pm$ $20 \mathrm{pN}$. The solid curve shows results from a model calculation for a 96 -atom flake (see text). which, for modest loads, can be attributed to the elastic variation in the contact area with the load [21]. In the present measurements on graphite, the typical increase in friction between loads of 0 and $25 \mathrm{nN}$ was as low as $0.05 \%-0.4 \%$. This strongly suggests that the contact area was as good as constant over this force range, consistent with the presence of a flake with a finite, loadindependent area of contact to the substrate. Additional evidence for the presence of a graphite flake between the tip and the substrate comes from a set of independent large-scale measurements on a polycrystalline graphite sample (grade ZYH), which showed substantial differences between the friction forces on neighboring grains. Because the lattice orientation is different on every grain, the degree of incommensurability changes each time the flake is moved across a grain boundary, leading to an abrupt change in the friction. We have also observed on some occasions that, after a deliberate, modest misalignment by a few degrees of an initially aligned contact, the high friction restored spontaneously in the course of a few scan lines. We interpret these events as the effect of the torque exerted by the surface on a nearly aligned flake, which was attached to the tungsten tip sufficiently loosely to rotate. In fact, reproducible measurements, such as those in Fig. 3, in which the same relative orientations always led to the same friction loops, were quite rare, the fixed-flake situation occurring as the exception, rather than the rule. Nevertheless, all observations resemble either those in Figs. 2(a) and 2(b) or those in Figs. 2(c)-2(e); i.e., all measurements were consistent with friction between a graphite flake and the graphite substrate.

We have used high-resolution TEM to image the tungsten tip after our friction experiments. The TEM analysis showed that the tungsten tip had a radius of about $80 \mathrm{~nm}$ and was covered with a smooth amorphous layer of $7 \mathrm{~nm}$ thick tungsten oxide. Unfortunately, thorough TEM inspection of the tip was not possible due to rapid removal of the amorphous layer by the electron beam. As a result, no clear TEM images could be obtained of either a multilayer flake or a single layer of graphite, although some weak features were present, suggestive of multilayers with the graphite lattice spacing.

The peak width in Fig. 3 directly reflects the flake diameter. For example, a single-atom tip should show high friction for all orientations, while an infinitely large contact would be completely superlubric, except for infinitely narrow angular ranges around perfect registry, if we assume the flake and the substrate to be completely rigid. For finite-size contacts, the cancellation of lateral forces, which causes superlubricity, can be considered complete when the mismatch between the two lattices adds up to one lattice spacing over the diameter of the contact. This mismatch condition provides us with the estimate that $\tan (\Delta \theta)=1 / D$, where $\Delta \theta$ is the full width at half maximum of the friction peak, and $D$ is the flake 
diameter, expressed in lattice spacings. From the widths in Fig. 2 , of $5.4^{\circ} \pm 1^{\circ}$ for the first peak and $6.5^{\circ} \pm 0.8^{\circ}$ for the second, we estimate a flake diameter of 7 to 12 lattice spacings. How good this estimate is, is demonstrated by the excellent fit to the friction data in Fig. 3, provided by a modified Tomlinson model. In this model, we moved a rigid graphite flake, connected to the Tribolever springs, in a rigid hexagonal potential, reflecting the periodicity of the graphite surface. For a symmetric flake of 96 atoms and a potential depth of $5.5 \mathrm{meV}$ per atom, the model yielded peaks with a width of $5.5^{\circ}$ and a height of $268 \mathrm{pN}$, very similar to the experimental observations.

Other graphitic systems have been found that show remarkable nanotribological properties, which might be attributed to superlubricity. Our results might explain the very low interwall friction between nested carbon nanotubes (CNT), observed in TEM experiments [22]. In most cases, the inner and outer tubes in a multiwall CNT form an incommensurate graphitic system, similar to a rotated flake that slides over a graphite surface. Recently, the transition from sliding motion to rotation of CNTs on graphite was also considered to be caused by changes in the commensurability [23]. In contrast to our singlecontact FFM experiment, macroscopic-scale friction involves multiple microcontacts with different sizes and orientations. Based on our observations, one may speculate that in the case of macroscopic lubrication by graphite a large fraction of the graphite-graphite contacts will be in the "superlubric state," while only a small fraction will be in registry. This should lead to a tremendous reduction in the average friction force, experienced in the ensemble of microcontacts, and thus might explain the excellent lubrication by graphite and similar, layered materials, such as $\mathrm{MoS}_{2}$ and $\mathrm{Ti}_{3} \mathrm{SiC}_{2}$. In addition, indications that also in a macroscopic sliding contact of lamellar solids rotated flakes are created come from transmission electron microscopy (TEM) observations by Martin et al. [24] on $\mathrm{MoS}_{2}$. Finally, it has been shown that on top of diamondlike carbon coatings a graphitic tribolayer is formed under sliding conditions, which leads to a decrease of the friction coefficient after run-in [25]. Therefore, also the excellent friction properties of diamondlike carbon coatings might be caused by superlubric graphite contacts. We suppose that, for sufficiently large contacts, superlubricity might break down, as the two lattices are not perfectly rigid, and a network of misfit dislocations should form between the two, the motion of which will dissipate energy.

This work is part of the research program of the "Stichting voor Fundamenteel Onderzoek der Materie
(FOM)" and was made possible by financial support of the "Nederlandse Organisatie voor Wetenschappelijk Onderzoek (NWO)."

*Present address: IAVF Antriebstechnik AG, Im Schlehert 32, 76187 Karlsruhe, Germany.

†Electronic address: frenken@ phys.leidenuniv.nl

*Present address: Johns Hopkins Applied Physics Laboratory, 11100 Johns Hopkins Road, Laurel, MD 20723-6099, USA.

[1] W. Bragg, An Introduction to Crystal Analysis (Bell, London, 1928), p. 64.

[2] C. M. Mate, G. M. McClelland, R. Erlandsson, and S. Chiang, Phys. Rev. Lett. 59, 1942 (1987).

[3] J.-A. Ruan and B. Bhushan, J. Mater. Res. 8, 3019 (1993).

[4] U. D. Schwarz, O. Zwörner, P. Köster, and R. Wiesendanger, Phys. Rev. B 56, 6987 (1997).

[5] E. Liu, B. Blanpain, J.-P. Celis, and J. R. Roos, J. Appl. Phys. 84, 4859 (1998).

[6] R. Buzio, E. Gnecco, C. Boragno, and U. Valbusa, Carbon 40, 883 (2002).

[7] G. A. Tomlinson, Philos. Mag. 7, 905 (1929).

[8] H. Hölscher, U. D. Schwarz, O. Zwörner, and R. Wiesendanger, Phys. Rev. B 57, 2477 (1998).

[9] N. Sasaki, K. Kobayashi, and M. Tsukada, Phys. Rev. B 54, 2138 (1996).

[10] N. Sasaki et al., Phys. Rev. B 57, 3785 (1998).

[11] M. Salmeron, in Surface Diagnostics in Tribology, edited by K. Miyoshi and Y.W. Chung (World Scientific, Singapore, 1993), p. 75.

[12] M. Hirano and K. Shinjo, Phys. Rev. B 41, 11837 (1990).

[13] K. Shinjo and M. Hirano, Surf. Sci. 283, 473 (1993).

[14] M. R. Sørensen, K. W. Jacobsen, and P. Stoltze, Phys. Rev. B 53, 2101 (1996).

[15] M. Hirano, K. Shinjo, R. Kaneko, and Y. Murata, Phys. Rev. Lett. 67, 2642 (1991).

[16] M. Hirano, K. Shinjo, R. Kaneko, and Y. Murata, Phys. Rev. Lett. 78, 1448 (1997).

[17] J. S. Ko and A. J. Gellman, Langmuir 16, 8343 (2000).

[18] T. Zijlstra et al., Sensors Actuators A 84, 18 (2000).

[19] J. P. Cleveland, S. Manne, D. Bocek, and P. K. Hansma, Rev. Sci. Instrum. 64, 403 (1993).

[20] J. Wang, K. C. Rose, and C. M. Lieber, J. Phys. Chem. B 103, 8405 (1999)

[21] R.W. Carpick, N. Agraït, D. F. Ogletree, and M. Salmeron, J. Vac. Sci. Technol. B 14, 1289 (1996).

[22] J. Cumings and A. Zettl, Science 289, 602 (2000).

[23] M. R. Falvo, J. Steele, R. M. Taylor II, and R. Superfine, Phys. Rev. B 62, R10 665 (2000).

[24] J. M. Martin, C. Donnet, T. LeMogne, and T. Epicier, Phys. Rev. B 48, 10583 (1993).

[25] Y. Liu, A. Erdemir, and E. I. Meletis, Surf. Coat. Technol. 86-87, 564 (1996). 